\title{
FundAMENTAL SETS OF FUNCTIONS ON SPHERES
}

\author{
V. A. Menegatto
}

\begin{abstract}
Let $S^{m}$ be the unit sphere in $R^{m+1}$ and let $\langle\cdot, \cdot\rangle$ denote the usual inner product in $\mathbb{R}^{m+1}$. We characterize those functions $K$ in $L^{p}([-1,1]), p \geq 1$, for which the associated set of zonal functions $\left\{x \in S^{m} \rightarrow K(\langle x, y\rangle): y \in S^{m}\right\}$ is fundamental in $L^{p}\left(S^{m}\right)$. We then study fundamentality of sets generated by either spherical convolution or spherical shifting, thus providing methods of construction of fundamental sets in $L^{p}\left(S^{m}\right)$.
\end{abstract}

\section{Introduction}

Let $S^{m}$ be the unit sphere in the Euclidean space $\mathbb{R}^{m+1}$ and let $\langle\cdot, \cdot\rangle$ denote the usual inner product in $\mathbb{R}^{m+1}$. In this paper, we study approximations of a given function or a class of functions defined on $S^{m}$ by linear combinations of functions of the form

$$
x \in S^{m} \longrightarrow K(\langle x, y\rangle), \quad y \in S^{m} \text {. }
$$

Following the literature, we call any function of the form (1.1) a $y$-zonal (spherical) function, or a zonal function on $S^{m}$ for short. A zonal function is the spherical counterpart of what is called a radial function in $\mathbb{R}^{m}$. As we shall see, the approximation can be done even when all the zonal functions are constructed from a fixed function $K$. Hence, some choices of $K$ will not only serve for approximation purposes but also for solving scattered data interpolation problems on spheres (see [4] for details on this).

Our starting point is reference [12] where the continuous functions $K$, defined on $[-1,1]$ for which the set

$$
\mathcal{M}(K):=\left\{x \in S^{m} \longrightarrow K(\langle x, y\rangle): y \in S^{m}\right\}
$$

is fundamental in the space $C\left(S^{m}\right)$ of all continuous functions on $S^{m}$, were completely identified. There, the space $C\left(S^{m}\right)$ was assumed (and we do the same here) to be endowed with the topology of uniform convergence. Recall that a subset $\mathcal{F}$ of a normed linear space $V$ is fundamental (total) in $V$ if its linear span is dense in $V$. We observe that the set $\mathcal{M}(K)$ obviously depends on $m$, even though our notation neglects that fact. The proof of the result in [12] was achieved via another one, not easily found in the literature, concerning the uniform Cèsaro $(C, m)$ summability of the formal Fourier expansion of $K$ in terms of Gegenbauer polynomials. A shorter proof of the result in [12] was given later in [9].

In the present paper, we first extend the result in [12] to the $L^{p}$ context. More precisely, we identify the functions $K$ for which the set $\mathcal{M}(K)$ is fundamental in $L^{p}\left(S^{m}\right)$. In this context, the definition of $\mathcal{M}(K)$ may be relaxed so that $y$ may vary in a subset

Received 26 November 1997, revised 25 August 1998.

1991 Mathematics Subject Classification: 41A30, 41A45, 41A63, 42A65, 42A82, 42C10, $42 \mathrm{C} 30$.

Key words and phrases: fundamentality, approximation on spheres, spherical harmonics, spherical convolution, spherical shifting, zonal functions. 
of $S^{m}$ whose complement is negligible and $K$ may not be continuous. Our method of proof is not related to that of the result in [12] but has some resemblance to the one in [9]. This is done in Section 2, after a brief review of $L^{p}$ spaces and spherical harmonics. In Section 3, after listing some properties of spherical convolution, we apply the results and ideas from Section 1 to examine fundamentality of sets generated by such convolution. Some of the results in this section hold for general sets of functions rather than sets of zonal functions. In Section 4, we repeat the steps of Section 3, now considering sets generated by spherical shifting.

\section{Fundamental sets in $L^{p}\left(S^{m}\right)$}

Let $d w_{m}$ be the standard surface measure on $S^{m}$ so that

$$
w_{m}=\int_{S^{m}} d w_{m}=\frac{2 \pi^{m / 2}}{\Gamma(m / 2)} .
$$

A real-valued $w_{m}$-measurable function $f$ defined on $S^{m}$ is called $p$-integrable $(p \geq 1)$ if

$$
\|f\|_{p}:=\left(\frac{1}{w_{m}} \int_{S^{m}}|K(x)|^{p} d w_{m}(x)\right)^{1 / p}<\infty .
$$

We shall denote by $L^{p}\left(S^{m}\right)$ the vector space consisting of $p$-integrable functions defined on $S^{m}$ where we consider that two functions $f$ and $g$ are equal in $L^{p}\left(S^{m}\right)$ whenever $\|f-g\|_{p}=0$. The most important of these spaces is $L^{2}\left(S^{m}\right)$. It is a Hilbert space with inner product given by

$$
\left\langle\langle f, g\rangle:=\frac{1}{w_{m}} \int_{S^{m}} f(x) g(x) d w_{m}(x), \quad f, g \in L^{2}\left(S^{m}\right) .\right.
$$

However, this nice structure will not be exploited in this paper. A remark concerning our notation is that both the norm and the inner product above depend on $m$, but we do not enforce that fact.

It also is convenient to introduce the space $L^{\infty}\left(S^{m}\right)$ but the reader is advised that almost all results in this paper do not hold for the case $p=\infty$. It is composed of all functions defined on $S^{m}$ which are $w_{m}$-measurable and $w_{m}$-essentially bounded. The reader is invited to consult Chapter $\mathrm{V}$ in [6] for more details on this definition.

Spherical harmonics are the main tool in the proofs of our results. We recall some basic facts about them but we assume that the reader is familiar with this topic and, therefore, the results are stated without proof. General information on spherical harmonics, including proofs of the results used here can be found in $[8,11,13,14]$. A spherical harmonic of degree $k$ in $m+1$ variables is the restriction to $S^{m}$ of a homogeneous harmonic polynomial on $\mathbb{R}^{m+1}$ of degree $k$. The space of spherical harmonics of degree $k$ in $m+1$ variables is denoted by $\mathcal{H}_{k}^{m+1}$, and its finite dimension is denoted by $N_{k}^{m}$. If $k \neq l$, then $\mathcal{H}_{k}^{m}$ and $\mathcal{H}_{l}^{m}$ are orthogonal with respect to the inner product above. The restriction to $S^{m}$ of a polynomial on $\mathbb{R}^{m+1}$ of degree at most $j$ is an element of $\bigoplus_{k=0}^{j} \mathcal{H}_{k}^{m}$. This observation and the fact that polynomials on $\mathbb{R}^{m+1}$ are dense in the space $C\left(S^{m}\right)$ gives rise to the following important result (see either p. 222 in [13] or p. 448 in [14]).

Lemma 2.1. The set $\bigcup \mathcal{H}_{k}^{m}$ is fundamental in $C\left(S^{m}\right)$. 
Another interesting property about spherical harmonics is the so-called Funk-Hecke formula. Before stating it, we need some additional notation. Hereafter, if $p \geq 1$, we write $L^{p, m}([-1,1])$ to denote the space of all functions defined in $[-1,1]$ which are $p$-integrable with respect to the measure $d r_{m}:=\left(1-t^{2}\right)^{(m-2) / 2} d t$. Thus, a function $K$ is in $L^{p, m}([-1,1])$ if and only if

$$
\|K\|_{p, m}:=\left(\frac{w_{m-1}}{w_{m}} \int_{-1}^{1}|K(t)|^{p}\left(1-t^{2}\right)^{(m-2) / 2} d t\right)^{1 / p}<\infty
$$

and we identify functions $K$ and $L$ for which $\|K-L\|_{p, m}=0$. Given a function $K$ in some $L^{p, m}([-1,1])$, the coefficients in its formal Fourier expansion

$$
K(t) \sim \sum_{k=0}^{\infty} a_{k}^{m}(K) P_{k}^{(m-1) / 2}(t)
$$

in terms of Gegenbauer polynomials, are calculated by

$$
a_{k}^{m}(K):=\frac{w_{m-1}}{w_{m}} \int_{-1}^{1} K(t) p_{k}^{(m-1) / 2}(t)\left(1-t^{2}\right)^{(m-2) / 2} d t, \quad k=0,1,2, \ldots
$$

where $p_{k}^{(m-1) / 2}=P_{k}^{(m-1) / 2} / P_{k}^{(m-1) / 2}(1)$.

The Funk-Hecke formula now reads as follows [11].

Lemma 2.2 (Funk-Hecke). For any $K$ in $L^{1, m}([-1,1])$ and any $Y_{k}^{m}$ in $\mathcal{H}_{k}^{m}$,

$$
\frac{1}{w_{m}} \int_{S^{m}} K(\langle x, y\rangle) Y_{k}^{m}(x) d w_{m}(x)=a_{k}^{m}(K) Y_{k}^{m}(y), \quad y \in S^{m} .
$$

The spaces $L^{p, m}([-1,1])$ and $L^{p}\left(S^{m}\right)$ are connected to each other in a standard way. If $K$ is in $L^{p, m}([-1,1])$, then $K(\langle\cdot, y\rangle)$ is in $L^{p}\left(S^{m}\right)$ for all $y \in S^{m}$. Conversely, if $K(\langle\cdot, y\rangle)$ is in $L^{p}\left(S^{m}\right)$ for some $y \in S^{m}$, then $K$ is in $L^{p, m}([-1,1])$. Since $w_{m}<\infty$, Theorem IV-13.17 in [6] says that $L^{p}\left(S^{m}\right) \subset L^{1}\left(S^{m}\right), p \geq 1$. This fact, taken in conjunction with the above comments, immediately implies that each function $K(\langle\cdot, y\rangle)$, $y \in S^{m}$, is in $L^{1}\left(S^{m}\right)$, whenever $K$ is in $L^{p, m}([-1,1])$.

Before we describe our main results, we digress to quote a consequence of the HahnBanach Theorem related to fundamentality of sets in normed linear spaces. This result is well known, and we include it as a separate lemma due to its frequent use.

Lemma 2.3. Let $\mathcal{F}$ be a subset of a normed linear space $V$. In order that $\mathcal{F}$ be fundamental in $V$, it is necessary and sufficient that $\mathcal{F}$ not be annihilated by a nonzero bounded linear functional on $V$.

We now can state and prove the main result of this section.

Theorem 2.4. Let $K$ be in $L^{p, m}([-1,1])$. In order that the set $\mathcal{M}(K)$ be fundamental in $L^{p}\left(S^{m}\right)$, it is necessary and sufficient that $a_{k}^{m}(K) \neq 0, k=0,1,2, \ldots$.

Proof. We first prove that the condition is sufficient. Let $\mathcal{L}$ be a bounded linear functional on $L^{p}\left(S^{m}\right)$ which annihilates $\mathcal{M}(K)$. Using the Riesz representation theorem [6], we write $\mathcal{L}$ in the form $\mathcal{L}(g)=\left\langle\langle g, h\rangle, g \in L^{p}\left(S^{m}\right)\right.$, in which $h \in L^{q}\left(S^{m}\right)$, $1 / p+1 / q=1$ (as usual, $q=\infty$ whenever $p=1$ ). The annihilating property of $\mathcal{L}$ then reduces to

$$
\frac{1}{w_{m}} \int_{S^{m}} K(\langle x, y\rangle) h(x) d \omega_{m}(x)=0, \quad y \in S^{m} \text { a.e. }
$$


Next, we multiply both sides of the previous equality by $Y_{k}^{m}(y)$, and we integrate with respect to the surface measure $w_{m}$. Holder's inequality implies that $K(\langle x, y\rangle) h(x)$ $Y_{k}^{m}(y)$ is $w_{m} \times w_{m}$-integrable over $S^{m} \times S^{m}$, and hence, using Fubini's Theorem to interchange the order of integration, we obtain

$$
\frac{1}{w_{m}} \int_{S^{m}} h(x)\left(\int_{S^{m}} K(\langle x, y\rangle) Y_{k}^{m}(y) d w_{m}(y)\right) d \omega_{m}(x)=0,
$$

$Y_{k}^{m} \in \mathcal{H}_{k}^{m}, k=0,1,2, \ldots$ Using the Funk-Hecke formula, we reach

$$
a_{k}^{m}(K)\left\langle\left\langle h, Y_{k}^{m}\right\rangle\right\rangle=0, \quad Y_{k}^{m} \in \mathcal{H}_{k}^{m}, \quad k=0,1,2, \ldots
$$

From our hypotheses, it follows that

$$
\left\langle\left\langle h, Y_{k}^{m}\right\rangle\right\rangle=0, \quad Y_{k}^{m} \in \mathcal{H}_{k}^{m}, \quad k=0,1,2, \ldots
$$

That is, $\mathcal{L}$ annihilates $\cup \mathcal{H}_{k}^{m}$. Since $C\left(S^{m}\right)$ is dense in $L^{p}\left(S^{m}\right)$ (Theorem IV-13.21 in [6]), Lemma 2.1 implies that $\cup \mathcal{H}_{k}^{m}$ is fundamental in $L^{p}\left(S^{m}\right)$. Thus, by Lemma 2.3, $\mathcal{L}=0$. Therefore, $\mathcal{M}(K)$ is fundamental in $L^{p}\left(S^{m}\right)$ by Lemma 2.3 .

Conversely, assume that there is an index $l$ such that $a_{l}^{m}(K)=0$. Select $Y_{l}^{m} \in \mathcal{H}_{l}^{m}$ and consider the nonzero Borel measure $\lambda$ on $S^{m}$ defined on the family $\mathcal{B}$ of Borel subsets of $S^{m}$ by the following integral:

$$
\lambda(B):=\int_{B} Y_{l}^{m} d w_{m}, \quad B \in \mathcal{B} .
$$

For any fixed $y$, we may use Lemma 2.2 once again to obtain

$$
\begin{aligned}
\int_{S^{m}} K(\langle x, y\rangle) d \lambda(x) & =\int_{S^{m}} K(\langle x, y\rangle) Y_{l}^{m}(x) d w_{m}(x) \\
& =w_{m} a_{l}^{m}(K) Y_{l}^{m}(y)=0 .
\end{aligned}
$$

Thus, the nontrivial bounded linear functional $\mathcal{L}_{1}$ over $L^{p}\left(S^{m}\right)$ given by $\mathcal{L}_{1}(g):=$ $\int_{S^{m}} g d \lambda$ annihilates $\mathcal{M}(K)$. By Lemma $2.3, \mathcal{M}(K)$ is not fundamental in $L^{p}\left(S^{m}\right)$.

We observe that the argument used in the second half of the proof of Theorem 2.4 already has been used in several papers (see [12], for example). What is different in our procedure is the use of the Funk-Hecke formula to tie things up.

Since orthogonal transformations of $\mathbb{R}^{m+1}$ can be regarded as coordinate transformations of $S^{m}$ which leave $d w_{m}$ unchanged, we have the result:

Corollary 2.5. Let $p, m$, and $K$ be as in the previous theorem. The following are equivalent:

(i) $\mathcal{M}(K)$ is fundamental in $L^{p}\left(S^{m}\right)$;

(ii) $\mathcal{M}(A, K):=\left\{x \in S^{m} \rightarrow K(\langle A x, y\rangle): y \in S^{m}\right.$ a.e. $\}$ is fundamental in $L^{p}\left(S^{m}\right)$ for some orthogonal transformation $A$ of $\mathbb{R}^{m+1}$; $\mathbb{R}^{m+1}$.

(iii) $\mathcal{M}(A, K)$ is fundamental in $L^{p}\left(S^{m}\right)$ for all orthogonal transformations $A$ of

Proof. This follows directly from the fact that $\mathcal{M}(A, K)=\mathcal{M}(K)$ whenever $A$ is an orthogonal transformation of $\mathbb{R}^{m+1}$.

It is a straightforward calculation to verify that every isometry of $S^{m}$ is the restriction of an orthogonal transformation of $R^{m+1}$. Thus, the previous corollary holds true for isometries and motions of $S^{m}$. 
We close this section by presenting another elementary consequence of the proof of Theorem 2.4. Its proof and the formulation of possible extensions are left to the reader.

Corollary 2.6. Let $K_{1}$ and $K_{2}$ be functions in $L^{p, m}([-1,1])$. In order that the set $\mathcal{M}\left(K_{1}\right) \cup \mathcal{M}\left(K_{2}\right)$ be fundamental in $L^{p}\left(S^{m}\right)$, it is necessary and sufficient that $\left|a_{k}^{m}\left(K_{1}\right)\right|+\left|a_{k}^{m}\left(K_{2}\right)\right| \neq 0, k=0,1,2 \ldots$

If $K$ is either a strictly positive definite kernel or a nonnegative strictly conditionally negative definite kernel on the Hilbert sphere, then $a_{k}^{m}(K) \neq 0$ for all $k$. Hence, according to results in [7], $K$ can be used not only to generate approximants to any function in $L^{p}\left(S^{m}\right)$, but it also can be used to construct interpolants for solving scattered data interpolation problems on spheres. Certain completely monotonic functions naturally belong to these classes of functions [7].

Finally, note that the above results hold for complex-valued functions although we do not go into the details of that. Instead, we present a concrete example of a complex function $K$ that fits in our theorems. For a positive real number $r$, the function $K_{r}(t)=\exp (-i r t)$ is such that (see Chapter 7 in [1])

$$
a_{k}^{m}\left(K_{r}\right)=\frac{(-i)^{k} 2^{(m+1) / 2} \Gamma((m+1) / 2)}{r^{(m-1) / 2}} J_{k-1+(m+1) / 2}(r)
$$

where $\Gamma$ is the Gamma function and $J_{\alpha}$ is the Bessel function. If $r$ is such that $J_{k-1+(m+1) / 2}(r) \neq 0$ for all $k$, then $a_{k}^{m}\left(K_{r}\right) \neq 0$ for all $k$ as needed. It is well known that if $x_{\nu}$ is the smallest positive zero of $J_{\nu},(\nu>0)$, then $x_{\nu}>\nu$ (p. 981 in [5]). Since $J_{0}(1) \neq 0$, then $a_{k}^{m}\left(K_{1}\right) \neq 0$ for all $k$ and, therefore, $K_{1}$ is such an example.

\section{Fundamental sets by convolution}

In this section, we turn to consequences of Corollary 2.5 concerning the fundamentality of sets generated by spherical convolution. The precise nature of the results we deal with here is as follows. For an appropriate function $K$ in $L^{1, m}([-1,1])$ and a subset $\mathcal{F}$ of $L^{p}\left(S^{m}\right)$, we give conditions in order that the set $K * \mathcal{F}:=\{K * f: f \in \mathcal{F}\}$ be fundamental in $L^{p}\left(S^{m}\right)$. Recall that if $K \in L^{1, m}([-1,1])$ and if $f \in L^{p}\left(S^{m}\right)$, then the spherical convolution $K * f$ of $K$ and $f$ over $S^{m}$ is given by

$$
K * f(x):=\frac{1}{w_{m}} \int_{S^{m}} K(\langle x, y\rangle) f(y) d w_{m}(y), \quad x \in S^{m} \text { a.e. }
$$

Notice that spherical convolution is not commutative. So, another completely different problem is to begin with a function $f$ in $L^{p}\left(S^{m}\right)$ and a subset $\mathcal{K}$ of $L^{1, m}([-1,1])$ and then find conditions in order that the set $\mathcal{K} * f:=\{K * f: K \in \mathcal{K}\}$ be fundamental in $L^{p}\left(S^{m}\right)$. In this section, we discuss these two questions.

We collect some properties of spherical convolution in Lemma 3.1.

Lemma 3.1. Fix $1 \leq p, q<\infty$ and let $K$ be in $L^{q, m}([-1,1])$. If $f$ is in $L^{p}\left(S^{m}\right)$, then $K * f$ is in $L^{r}\left(S^{m}\right)$ whenever $r:=p q /(p+q-p q) \geq 1$. If, in addition, $f$ is a $\zeta$-zonal function, then $K * f$ is a $\zeta$-zonal function also.

Proof. The first claim of the lemma is essentially due to the well-known Young's inequality. For the second part, first observe that

$$
\int_{S^{1}} K(\langle x, y\rangle) f(\langle\zeta, y\rangle) d w_{1}(y)=\int_{0}^{\pi} K(\cos \phi) f(\cos (\phi-\psi)) \sin \phi d \phi, \quad x, \zeta \in S^{1}
$$


where $\psi$ is the angle between $x$ and $\zeta$. Thus, the second assertion of the lemma holds for $m=1$. To handle the case $m>1$, we use the well-known fact that a function $f$ defined on $S^{m}$ is $\zeta$-zonal if and only if $f \circ A=f$ for all orthogonal transformations of $\mathbb{R}^{m+1}$ fixing $\zeta$. Let $A$ be an orthogonal transformation of $\mathbb{R}^{m+1}$ fixing $\zeta$ and suppose that $f \circ A=f$. Then,

$$
\begin{aligned}
K * f(A x) & =\int_{S^{m}} K(\langle A x, y\rangle) f(y) d w_{m}(y) \\
& =\int_{S^{m}} K\left(\left\langle x, A^{-1} y\right\rangle\right) f(y) d w_{m}(y) \\
& =\int_{S^{m}} K(\langle x, y\rangle) f(A y) d w_{m}(y) \\
& =K * f(x) \quad x \in S^{m} \text { a.e. }
\end{aligned}
$$

The invariance of $d w_{m}$ with respect to orthogonal transformations of $\mathbb{R}^{m+1}$ has been used in the third equality above. Thus, $(K * f) \circ A=K * f$ as needed.

Our first result in this section is now at hand.

Theorem 3.2. Let $p, q$ be as in Lemma 3.1 and write $r=p q /(p+q-p q)$. Let $K$ be in $L^{q, m}([-1,1])$ and let $\mathcal{F}$ be a subset of $L^{p}\left(S^{m}\right)$. If $K * \mathcal{F}$ is fundamental in $L^{r}\left(S^{m}\right)$, then $a_{k}^{m}(K) \neq 0, k=0,1,2, \ldots$ Conversely, if the latter holds and $\mathcal{F}$ is fundamental in $L^{p}\left(S^{m}\right)$, then $K * \mathcal{F}$ is fundamental in $L^{r}\left(S^{m}\right)$.

Proof. For the first part, we assume that $a_{l}^{m}(K)=0$ for some $l$ and show that $K * \mathcal{F}$ is not fundamental in $L^{r}\left(S^{m}\right)$. Consider the measure $\lambda$ defined in the second half of the proof of Theorem 2.4. By integrating the elements of $K * \mathcal{F}$ with respect to that measure and using Lemma 2.2, we obtain

$$
\begin{aligned}
\left\langle\left\langle K * f, Y_{l j}^{m}\right\rangle\right\rangle & =\frac{1}{w_{m}^{2}} \int_{S^{m}} \int_{S^{m}} K(\langle x, y\rangle) f(y) Y_{l j}^{m}(x) d w_{m}(y) d w_{m}(x) \\
& =\frac{1}{w_{m}} \int_{S^{m}} f(y) K * Y_{l j}^{m}(y) d w_{m}(y) \\
& =a_{l}^{m}(K)\left\langle\left\langle f, Y_{l j}^{m}\right\rangle\right\rangle \\
& =0, \quad g \in \mathcal{F} .
\end{aligned}
$$

Thus, $K * \mathcal{F}$ is annihilated by a nonzero continuous linear functional over $L^{r}\left(S^{m}\right)$. Hence, $K * \mathcal{F}$ is not fundamental in $L^{r}\left(S^{m}\right)$ by Lemma 2.3 .

For the second part, we assume that $\mathcal{F}$ is fundamental in $L^{p}\left(S^{m}\right)$ and that $a_{k}^{m}(K) \neq$ $0, k=0,1,2, \ldots$, and we prove that the condition

$$
\langle\langle K * f, h\rangle\rangle=0, \quad f \in \mathcal{F},
$$

for some $h \in L^{s}\left(S^{m}\right), 1 / s+1 / r=1$, implies that $h=0$ a.e. Using the definition of convolution in the above expression, interchanging the order of integration, and arranging, we obtain

$$
\frac{1}{w_{m}} \int_{S^{m}} f(y) K * h(y) d w_{m}(y)=0, \quad f \in \mathcal{F} .
$$

Thus, combining the fundamentality of $\mathcal{F}$ in $L^{p}\left(S^{m}\right)$ with Lemma 2.3, we see that $K * h=0$ a.e. Repeating the procedure in the first half of the proof of Theorem 2.4, we achieve the required conclusion for $h$. 
The proof of the previous theorem can be made much shorter if certain properties of convolution, closely related to those of convolution of periodic functions, are available. The validation of one such property is already implicit in the above proof. We formally quote it in the proof of the following result:

Corollary 3.3. Let $p, q, r, K$, and $\mathcal{F}$ be as in the previous theorem. If $K * \mathcal{F}$ is fundamental in $L^{r}\left(S^{m}\right)$, then the following condition must hold: if $f$ is in $\mathcal{F}$ and $\left\langle\left\langle K * f, Y^{m}\right\rangle\right\rangle=0$ for some $Y^{m}$ in $\bigcup_{k=0}^{\infty} \mathcal{H}_{k}^{m}$, then $\left\langle\left\langle f, Y^{m}\right\rangle\right\rangle=0$.

Proof. It suffices to observe that the proof of Theorem 3.2 gives the following property for $K$ and $\mathcal{F}$ :

$$
\left\langle K * f, Y_{k}^{m}\right\rangle=a_{k}^{m}(K)\left\langle\left\langle f, Y_{k}^{m}\right\rangle\right\rangle, \quad f \in \mathcal{F}, \quad Y_{k}^{m} \in \mathcal{H}_{k}^{m} .
$$

Now, if $K * \mathcal{F}$ is fundamental in $L^{r}\left(S^{m}\right)$, then $a_{k}^{m}(K) \neq 0$ for all $k$ by the previous theorem. Therefore, if the left-hand side of the above equality vanishes for some $f$, the same is true of $\left\langle\left\langle f, Y_{k}^{m}\right\rangle\right\rangle$.

Similarly, we have the following result.

Theorem 3.4. Let $p, q$, and $r$ be as before. Let $\mathcal{K}$ be a subset of $L^{q, m}([-1,1])$ and $f$ a function in $L^{p}\left(S^{m}\right)$. If $\mathcal{K} * f$ is fundamental in $L^{r}\left(S^{m}\right)$, then the following conditions hold:

(i) $\left\langle\left\langle f, Y^{m}\right\rangle\right\rangle \neq 0$, for all $Y^{m}$ in $\bigcup_{k=0}^{\infty} \mathcal{H}_{k}^{m}$;

(ii) If $K \in \mathcal{K}$ and $\left\langle\left\langle K * f, Y^{m}\right\rangle\right\rangle=0$ for some $Y^{m}$ in $\bigcup_{k=0}^{\infty} \mathcal{H}_{k}^{m}$, then $a_{k}^{m}(K)=0$.

If $f$ is zonal, say $f(\cdot)=P(\langle\cdot, \zeta\rangle)$ for some $P$ and some $\zeta$, then it is easily seen that formula (3.2) reduces, via the Funk-Hecke formula, to

$$
a_{k}^{m}(K * f)=a_{k}^{m}(K) a_{k}^{m}(P), \quad k=0,1,2, \ldots
$$

Thus, in particular, spherical convolution becomes a commutative property when restricted to zonal functions. Formula (3.3) helps in the conclusion of our last result of this section.

Theorem 3.5. Let $p, q, r$, and $K$ be as before and let $P \in L^{p, m}([-1,1])$. The following are equivalent:

(i) $K * \mathcal{M}(P)$ is fundamental in $L^{r}\left(S^{m}\right)$;

(ii) $\mathcal{M}(K) * P$ is fundamental in $L^{r}\left(S^{m}\right)$;

(iii) $a_{k}^{m}(K) a_{k}^{m}(P) \neq 0, k=0,1,2, \ldots$.

Proof. It suffices to prove that (i) and (iii) are equivalent. If (i) holds, then, as in the proof of Theorem 3.2, we have that $a_{k}^{m}(K) \neq 0$ and $a_{k}^{m}(P) \neq 0$ for all $k$. Conversely, if (iii) holds, then $\mathcal{M}(P)$ is already fundamental in $L^{p}\left(S^{m}\right)$ by Theorem 2.4. Hence, (i) follows directly from Theorem 3.2.

\section{Fundamental sets by shifting}

In this section, we study fundamentality of sets generated by what we call spherical shifting. The notion of shifting used here was apparently introduced by Rudin in [10], but for the case $m=2$ only. Later, this concept was further explored in [2] in connection with the study of saturation problems on spheres. More recently it has reappeared as an important ingredient in the definition of several moduli of smoothness 
of functions defined on spheres (see [3] and references therein). For $t$ in $(-1,1)$, the spherical shifting by $t$ of $f$ over $S^{m}$ is given by the following averaging process:

$$
S_{t}^{m}(f)(x):=\frac{1}{w_{m-1}\left(1-t^{2}\right)^{(m-1) / 2}} \int_{\langle x, y\rangle=t} f(y) d y, \quad x \in S^{m}
$$

where $d y$ is the measure element of the spherical section $\left\{y \in S^{m}:\langle x, y\rangle=t\right\}$. Thus, $S_{t}^{m}(f)(x)$ can be interpreted as the mean value of $f$ on the surface of an $m$-dimensional sphere of radius $\left(1-t^{2}\right)^{1 / 2}$. Since each $y$ in the spherical section can be represented in the form

$$
y=x t+z\left(1-t^{2}\right)^{1 / 2}, \quad\langle z, x\rangle=0
$$

a change of variables can be used to reduce the above integral to the form

$$
S_{t}^{m}(f)(x)=\frac{1}{w_{m-1}} \int_{S^{m-1}} f\left(x t+z\left(1-t^{2}\right)^{1 / 2}\right) d w_{m-1}(z), \quad x \in S^{m} .
$$

Among several properties of the operator $S_{t}^{m}$, we mention that it maps each space considered in this paper into itself and, in each case, it has norm 1. Proofs of these properties along with more information on this operator can be found in [2]. Perhaps the most important of its properties is the following:

$$
S_{t}^{m}\left(Y_{k}^{m}\right)=p_{k}^{(m-1) / 2}(t) Y_{k}^{m}, \quad t \in(-1,1), \quad Y_{k}^{m} \in \mathcal{H}_{k}^{m}, \quad k=0,1,2, \ldots
$$

One way of proving this is to combine the so-called addition formula for Gegenbauer polynomials (p. 472 in [14]) with the fact that for every $k$ and every $Y_{k}^{m}$ in $\mathcal{H}_{k}^{m}$, there are $\zeta_{1}, \zeta_{2}, \ldots, \zeta_{N_{k}^{m}}$ in $S^{m}$ and real numbers $c_{1}, c_{2}, \ldots, c_{N_{k}^{m}}$ such that

$$
Y_{k}^{m}(x)=\sum_{j=1}^{N_{k}^{m}} c_{j} p_{k}^{(m-1) / 2}\left(\left\langle x, \zeta_{j}\right\rangle\right), \quad x \in S^{m}
$$

As a consequence of (4.1), we have [2]:

Lemma 4.1. Let $f$ be a function in $L^{p}\left(S^{m}\right)$. Then, for any $t$ in $(-1,1)$,

$$
\left\langle\left\langle S_{t}^{m}(f), Y_{k}^{m}\right\rangle\right\rangle=p_{k}^{(m-1) / 2}(t)\left\langle\left\langle f, Y_{k}^{m}\right\rangle\right\rangle, \quad Y_{k}^{m} \in \mathcal{H}_{k}^{m}, \quad k=0,1,2, \ldots
$$

Our first result in this section is a simple consequence of this lemma.

Theorem 4.2. Let $\mathcal{F}$ be a subset of $L^{p}\left(S^{m}\right)$. If $S_{t}^{m}(\mathcal{F})$ is fundamental in $L^{p}\left(S^{m}\right)$ for some $t$ in $(-1,1)$, then the following conditions hold:

(i) $P_{k}^{(m-1) / 2}(t) \neq 0$, for $k=0,1,2, \ldots$; 0 .

(ii) If $f$ is in $\mathcal{F}$ and $\left\langle\left\langle S_{t}^{m}(f), Y^{m}\right\rangle\right\rangle=0$, for some $Y^{m}$ in $\bigcup_{k=0}^{\infty} \mathcal{H}_{k}^{m}$, then $\left\langle\left\langle f, Y^{m}\right\rangle\right\rangle=$

Proof. If $P_{k}^{(m-1) / 2}(t)=0$ for some $k$, then Lemma 4.1 implies that the nonzero functional $\mathcal{L}$ over $L^{p}\left(S^{m}\right)$ given by $\mathcal{L}(g)=\left\langle\left\langle g, Y_{k}^{m}\right\rangle\right\rangle$ annihilates $S_{t}^{m}(\mathcal{F})$. Hence, the latter would not be fundamental in $L^{p}\left(S^{m}\right)$ by Lemma 2.3. That condition (ii) is necessary now follows immediately from this.

We do not know whether both the fundamentality of $\mathcal{F}$ in $L^{p}\left(S^{m}\right)$ and condition (i) in Theorem 4.2 ensure the fundamentality of $S_{t}^{m}(\mathcal{F})$. As a matter of fact, in Theorem 4.6 ahead, we will answer this question in the affirmative when $\mathcal{F}$ is of the form $\mathcal{M}(K)$ for some $K$.

Sets composed of shiftings of a single function may be fundamental. Using the same arguments of the above proof, we can easily obtain the following result. 
Theorem 4.3. Let $f$ be in $L^{p}\left(S^{m}\right)$. If $\left\{S_{t}^{m}(f): t \in(-1,1)\right\}$ is fundamental in $L^{p}\left(S^{m}\right)$, then the following conditions hold:

(i) $\left\langle\left\langle f, Y^{m}\right\rangle\right\rangle \neq 0$, for all $Y^{m}$ in $\bigcup_{k=0}^{\infty} \mathcal{H}_{k}^{m}$; 0 .

(ii) Ift is in $(-1,1)$ and $\left\langle\left\langle S_{t}^{m}(f), Y_{k}^{m}\right\rangle\right\rangle=0$ for some $Y_{k}^{m}$ in $\mathcal{H}_{k}^{m}$, then $P_{k}^{(m-1) / 2}(t) \neq$

We now return to zonal functions.

Lemma 4.4. If $f$ is a $\zeta$-zonal function, then so is $S_{t}^{m}(f)$.

Proof. Take an orthogonal transformation $A$ of $\mathbb{R}^{m+1}$ for which $A \zeta=\zeta$. Then, first using the fact that $f$ is $\zeta$-zonal and then the invariance of $d w_{m-1}$ under $A^{-1}$, we have that

$$
\begin{aligned}
S_{t}^{m}(f)(A x) & =\frac{1}{w_{m-1}} \int_{S^{m-1}} f\left(A x t+z\left(1-t^{2}\right)^{1 / 2}\right) d w_{m-1}(z) \\
& =\frac{1}{w_{m-1}} \int_{S^{m-1}} f\left(x t+A^{-1} z\left(1-t^{2}\right)^{1 / 2}\right) d w_{m-1}(z) \\
& =S_{t}^{m}(f)(x), \quad x \in S^{m} .
\end{aligned}
$$

Thus, $S_{t}^{m}(f) \circ A=S_{t}^{m}$ and, therefore, $S_{t}^{m}(f)$ is $\zeta$-zonal.

From now on, if $f(\cdot)=K(\langle\cdot, \zeta\rangle)$ for some function $K$, we will write $S_{t}^{m}(K)$ to denote the function $K^{\prime}$ satisfying $S_{t}^{m}(f)(\cdot)=K^{\prime}(\langle\cdot, \zeta\rangle)$. The following lemma complements the previous one.

Lemma 4.5. Let $f$ and $K$ be as in the previous paragraph. Then,

$$
a_{k}^{m}\left(S_{t}^{m}(K)\right)=p_{k}^{(m-1) / 2}(t) a_{k}^{m}(K), \quad k=0,1,2, \ldots
$$

Proof. This follows from Lemma 4.1 as the following steps show.

$$
\begin{aligned}
a_{k}^{m}\left(S_{t}^{m}(K)\right) Y_{k}^{m}(x) & =\frac{w_{m-1}}{w_{m}} \int_{-1}^{1} K^{\prime}(t) p_{k}^{(m-1) / 2}(t)\left(1-t^{2}\right)^{(m-2) / 2} d t \\
& =\frac{1}{w_{m}} \int_{S^{m}} K^{\prime}(\langle\zeta, y\rangle) p_{k}^{(m-1) / 2}(\langle\zeta, y\rangle) d w_{m}(y) \\
& =\frac{1}{w_{m}} \int_{S^{m}} S_{t}^{m}(f)(y) p_{k}^{(m-1) / 2}(\langle\zeta, y\rangle) d w_{m}(y) \\
& =p_{k}^{(m-1) / 2}(t) \frac{1}{w_{m}} \int_{S^{m}} f(y) p_{k}^{(m-1) / 2}(\langle\zeta, y\rangle) d w_{m}(y) \\
& =p_{k}^{(m-1) / 2}(t) a_{k}^{m}(K) .
\end{aligned}
$$

In the above, we have used the fact that $p_{k}^{(m-1) / 2}(\langle\cdot, \zeta\rangle)$ is an element of $\mathcal{H}_{k}^{m}$.

Lemma 4.5 leads to the following nice result.

Theorem 4.6. Let $K$ be a function in $L^{p, m}([-1,1])$.

(i) If $t$ in $(-1,1)$ is such that $P_{k}^{(m-1) / 2}(t) \neq 0$ for all $k$ and $\mathcal{M}(K)$ is fundamental in $L^{p}\left(S^{m}\right)$, then the same is true of $\mathcal{M}\left(S_{t}^{m}(K)\right)$.

(ii) If $\mathcal{M}\left(S_{t}^{m}(K)\right)$ is fundamental in $L^{p}\left(S^{m}\right)$ for some $t$, then the same is true of $\mathcal{M}(K)$;

(iii) $\mathcal{M}\left(S_{t}^{m}(K)\right)$ is fundamental in $L^{p}\left(S^{m}\right)$ if and only if $P_{k}^{(m-1) / 2}(t) a_{k}^{m}(K) \neq 0$ for all $k$. 
Proof. If the assumptions in (i) hold, then Theorem 2.4 implies that $p_{k}^{(m-1) / 2}(t) a_{k}^{m}(K)$ $\neq 0$ for all $k$. Hence, by Lemma $4.5, a_{k}^{m}\left(S_{t}^{m}(K)\right) \neq 0$ for all $k$. Thus, (i) follows from Theorem 2.4. Part (ii) follows from Theorem 4.2 and Theorem 2.4. Part (iii) is a combination of both (i) and (ii).

Since $\left|p_{k}^{(m-1) / 2}(t)\right| \neq 1$ for $t$ in $(-1,1)$, the following criterion is apparent.

Theorem 4.7. Let $K$ be a function in $L^{p, m}([-1,1])$. These assertions are equivalent:

(i) $\mathcal{M}(K)$ is fundamental in $L^{p}\left(S^{m}\right)$;

(ii) For some $t$ in $(-1,1), \mathcal{M}\left(K+S_{t}^{m}(K)\right)$ is fundamental in $L^{p}\left(S^{m}\right)$;

(iii) For some $t$ in $(-1,1), \mathcal{M}\left(K-S_{t}^{m}(K)\right)$ is fundamental in $L^{p}\left(S^{m}\right)$.

We close this paper by proving a theorem related to the problem mentioned at the end of the first paragraph of Section 3. To do that, we rewrite formula (3.1) in the following way:

$$
K * f(x)=\frac{w_{m-1}}{w_{m}} \int_{-1}^{1} K(t) S_{t}^{m}(f)(x)\left(1-t^{2}\right)^{(m-2) / 2} d t, \quad x \in S^{m} .
$$

Using Fubini's Theorem, it is not difficult to see that if $K$ is in $L^{1, m}([-1,1]), f$ is in $L^{p}\left(S^{m}\right)$, and $h$ is in $L^{q}\left(S^{m}\right), 1 / p+1 / q=1$, then the following formula holds:

$$
\langle\langle K * f, h\rangle\rangle=\frac{w_{m-1}}{w_{m}} \int_{-1}^{1}\left\langle\left\langle S_{t}^{m}(f), h\right\rangle\right\rangle(t)\left(1-t^{2}\right)^{(m-2) / 2} d t .
$$

Implicit in this formula is the fact that the function

$$
t \longrightarrow\left\langle\left\langle S_{t}^{m}(f), h\right\rangle\right.
$$

is in $L^{p, m}([-1,1])$. One way of seeing this is as follows. Using the fact that the spherical shifting is an operator of norm 1, we have, by Holder's inequality, that

$$
\mid\left\langle\left\langle S_{t}^{m}(f), h\right\rangle\right| \leq\left\|S_{t}^{m}(f)\right\|_{p}\|h\|_{q} \leq\|f\|_{p}\|h\|_{q}, \quad t \in(-1,1),
$$

and so

$$
\begin{aligned}
\left\|\left\langle\left\langle S_{t}^{m}(f), h\right\rangle\right\rangle\right\|_{p, m} & \leq\|f\|_{p}\|h\|_{q} \int_{S^{m}}\left(1-t^{2}\right)^{(m-2) / 2} d t \\
& =\frac{w_{m}}{w_{m-1}}\|f\|_{p}\|h\|_{q} .
\end{aligned}
$$

Our final theorem is as follows.

Theorem 4.8. Let $\mathcal{K}$ be a subset of $L^{1, m}([-1,1])$ and $f$ a function in $L^{p}\left(S^{m}\right)$. If $\mathcal{K} * f$ is fundamental in $L^{p}\left(S^{m}\right)$, then $\left\{S_{t}^{m}(f): t \in(-1,1)\right\}$ is fundamental in $L^{p}\left(S^{m}\right)$. Conversely, if $\left\{S_{t}^{m}(f): t \in(-1,1) \backslash I\right\}$ is fundamental in $L^{p}\left(S^{m}\right)$ for every $r_{m}$-null subset $I$ of $[-1,1]$, and, in addition, $\mathcal{K}$ is fundamental in $L^{1, m}([-1,1])$, then $\mathcal{K} * f$ is fundamental in $L^{p}\left(S^{m}\right)$.

Proof. For the first part, we have to show that, under the given hypotheses, the condition

$$
\left\langle\left\langle S_{t}^{m}(f), h\right\rangle=0, \quad t \in(-1,1),\right.
$$

for some $h$ in $L^{q}\left(S^{m}\right), 1 / p+1 / q=1$, implies that $h=0$ a.e. To see that, observe that a combination of (4.2) and (4.3) yields

$$
\langle\langle K * f, h\rangle\rangle=0, \quad K \in \mathcal{K} .
$$


Our assumption and Lemma 2.3 then imply that $h=0$ a.e. For the second part, assume the hypotheses and suppose that

$$
\langle\langle K * f, h\rangle\rangle=0, \quad K \in \mathcal{K},
$$

for some $h$ as above. Formula (4.2) implies that

$$
\int_{-1}^{1}\left\langle\left\langle S_{t}^{m}(f), h\right\rangle\right\rangle K(t)\left(1-t^{2}\right)^{(m-2) / 2} d t=0, \quad K \in \mathcal{K} .
$$

The fundamentality of $\mathcal{K}$ in $L^{1, m}([-1,1])$ and Lemma 2.3 now imply that

$$
\left\langle\left\langle S_{t}^{m}(f), h\right\rangle=0, \quad t \in(-1,1) \backslash I,\right.
$$

for some $r_{m}$-null subset $I$ of $(-1,1)$. Finally, the fundamentality of

$$
\left\{S_{t}^{m}(f): t \in(-1,1) \backslash I\right\}
$$

in $L^{p}\left(S^{m}\right)$ together with Lemma 2.3 forces $h$ to be zero a.e.

Remark. Most of the results in Sections 3 and 4 may be restated for continuous functions. We believe this to be straightforward which is the reason why we have not done it here.

Acknowledgments. This work was completed while the author was visiting the Department of Mathematics of The University of Texas at Austin. We thank Professor E. W. Cheney who made this visit possible. The financial support from FAPESPBrazil (grant \# 96/4748-2) is gratefully acknowledged.

\section{References}

1. N. I. Akhiezer, Lectures on Integral Transforms, Translations of Mathematical Monographs, Vol. 70, American Mathematical Society, Providence, 1988.

2. H. Berens, P. L. Butzer, and S. Pawelke, Limitierungsverfahren von Riehen mehrdimen-sionaler Kugelfunktionen und deren Saturationsverhalten, Publ. Res. Inst. Math. Sci. Ser. A 4 (1968), 201-268.

3. H. Berens and L. Li, On the de la Vallee-Poussin means on the sphere, Results Math. 24 (1993), 12-26.

4. E. W. Cheney, Approximation using positive definite functions. In: Approximation Theory VIII (C. K. Chui and L. L. Schumaker, eds.), 1995, pp.1-24.

5. I. S. Gradshteiyn and I. M. Ryzhik, Tables of Integrals, Series, and Products, Academic Press, New York, 1980.

6. E. Hewitt and K. Stromberg, Real and Abstract Analysis, Graduate Texts in Mathemathics, Vol. 25, Springer-Verlag, New York, 1965.

7. V. A. Menegatto, Strictly positive definite kernels on the Hilbert sphere, Appl. Anal. 55 (1994), 91-101.

8. C. Müller, Spherical Harmonics, Lecture Notes in Mathematics, Vol. 17, Springer-Verlag, Berlin, 1966.

9. D. L. Ragozin and J. Levesley, The fundamentality of translates of zonal kernels on compact homogeneous spaces, preprint.

10. W. Rudin, Uniqueness theory for Laplace series, Trans. Amer. Math. Soc. 68 (1950), 287-303.

11. R. P. Seeley, Spherical harmonics, American Math. Monthly, 73 (1966), 115-121.

12. X. Sun and E. W. Cheney, Fundamental sets of continuous functions on spheres, Constr. Approx. 13 (1997), 245-250. 
13. M. Takeuchi, Modern Spherical Functions, Translations of Mathematical Monographs, Vol. 135, American Mathematical Society, Providence, 1991.

14. N. I. Vilenkin, Special Functions and the Theory of Group Representations, Translations of Mathematical Monographs, Vol. 22, American Mathematical Society, Providence, 1968.

ICMC-Universidade de SÃo PaUlo, 13560-970 SÃo Carlos SP, Brasil

E-mail: menegatt@icmsc.sc.usp.br 